\title{
Technical research on engine cycle constructing based on transformation model
}

\author{
$X i \mathrm{Hu}^{*}, \mathrm{Yu}$ Liu, and Xiaopan An \\ CATARC Automotive Test Center (Tianjin) Co.,Ltd, Tianjin 300300, China
}

\begin{abstract}
Keywords: WHSC, vehicle-engine transformation model, gear use strategy.

Abstract. This paper presents a technical method to derive engine test cycle by establishing a vehicle-to-engine cycle transform model ; Firstly, input, process and transform vehicle cycle and test vehicle data to get corresponding engine condition; Then, apply model built-in gear use strategy to select gear; Finally, under the selected gear, transform vehicle cycle into engine cycle termed by normalized speed and load. In addition, Comparison between model output cycle and WHTC cycle demonstrates that this transform method features consistency with present emission test standard, adaptability to various engine technologies and representative of real-life test scenarios.
\end{abstract}

\section{Introduction}

Type approval for exhaust emissions from heavy duty vehicles is generally conducted on an test bench where the engine is running under certain test cycle defining certain speed/load conditions on a second by second base. Engine cycle is important basis for government to enact vehicle emission test method and limitation standard, also for manufactures to research emission technique and develop products.

GB 17691-2019 Limits and measurement methods for exhaust pollutants from compression ignition and gas fueled positive ignition engines of vehicles (VI) (hereinafter referred to as 'N6') uses WHTC (World Harmonized Transient Cycle) as test cycle for emission type approve of heavy-duty vehicle engines. This cycle is constructed by transformation from WTVC (World Transit Vehicle Cycle) vehicle cycle. C-WTVC vehicle cycle presently used for China's heavy-duty vehicle fuel consumption type approval is a down-scale adaption of WTVC ${ }^{[1]}$.

Constructing engine cycle representative for various real-life heavy-duty engine operation can apply to technology development, match emissions in relative terms for accurate ranking of engines and technologies. Certain researches on engine cycle are carried out in China: Jiangsu University analyzed the correlation between test results and methods of vehicle and engine cycle ${ }^{[2]}$; Wuhan University of Technology established a neural network engine model for the dynamic emission prediction ${ }^{[3]}$. These researches have certain theoretical guiding and practical value for emission test. However, in the aspect of

\footnotetext{
* Corresponding author: huxi@catarc.ac.cn
} 
constructing engine cycle which can be used as the basis of vehicle development and evaluation, there are still lack of systematic research and substantive results.

Engine cycle constructing based on transformation from vehicle to engine is the natural advance for engine emission reduction technology. This paper presents a set of cycle constructing method by establishing an efficiently and convenient vehicle to engine cycle transformation model. Paper discusses the method in terms of data input and process, gear use calculates, cycle transformation and analysis. A light truck's data is applied to verify model's ability of providing technical support for model result.

\section{Outline of the constructing process}

The transformation model is composed of the several modules: data input and process module used to get necessary information of vehicle cycle, test vehicle and setting parameters; gear select module used to simulate the test gear-box and thus output gear use according to builtin strategy; transformation module used to calculate normalized speed and load factors on a second by second base, and finally output them as engine cycle result.

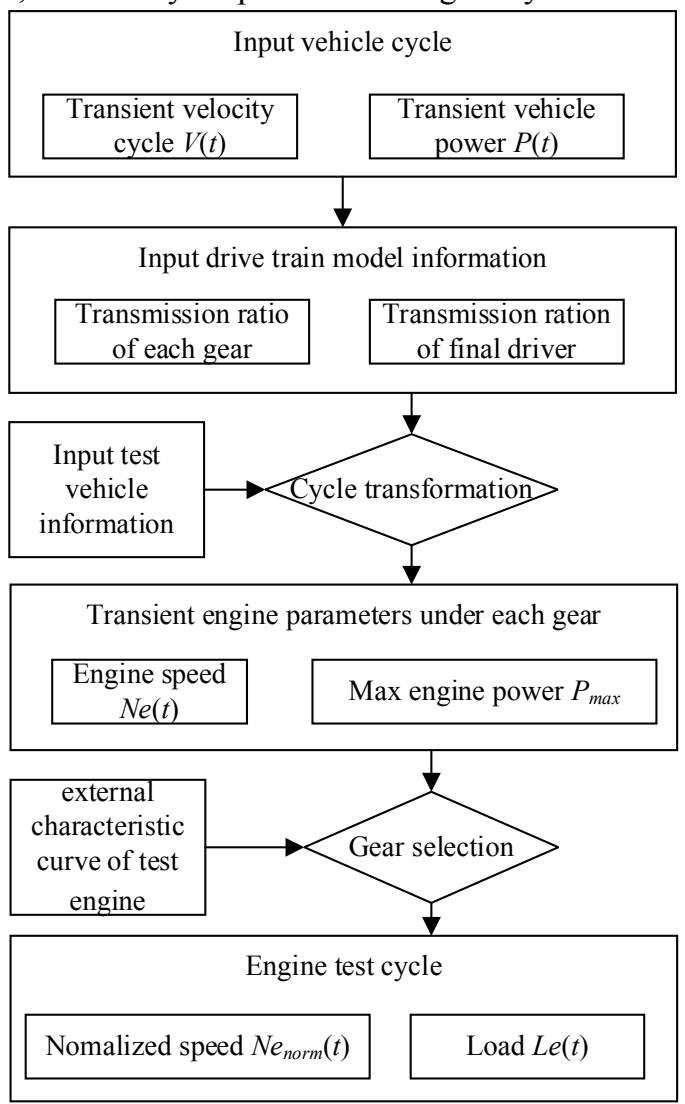

Fig. 1. Outline of cycle constructing process.

(1) Vehicle cycle

The vehicle cycle used in transformation is termed by transient vehicle velocity-time curve $V(t)$ and power-time curve $P(t)$. Input $V(t)$ and test vehicle data into the model, apply the driving resistance calculating formula in vehicle drum test to get the corresponding $P(t)$.

(2) Calculate characteristic engine speeds 
The external characteristic curve of the engine refers to the curve of the power or torque paired with engine speed at full load and serves as basis of gear selection. Input the EMP test information of the test engine into the model and calculate three important characteristic engine speeds: lowest speed $\left(n_{l o}\right)$, highest speed $\left(n_{h i}\right)$ and preferred speed $\left(n_{\text {pre }}\right)$.

(3) Gear selection

For every forward gear of the test vehicle, transform $V(t)$ to engine actual speed (rpm) time curve $N e(t)$ and normalize it into percentage form $N e_{\text {norm }}(\mathrm{t})$. Get maximum output engine power $P_{\max }(t)$ under each gear position from the external characteristic curve.

Combined with characteristic engine speeds, apply reasonable built-in gear use strategy to get the output gear second by second.

(4) Cycle output

Divide vehicle power $P(t)$ by maximum output power $P_{\max }(t)$ under the selected gear to get engine load - time curve in percentage form $L e(t)$; combined with $N e_{n o r m}(t)$ to output the test engine cycle.

\section{Cycle transformation example}

\subsection{Test vehicle information}

To demonstrate the transformation model, transformation was carried out using the representative vehicle cycle WTVC. Choose AUMARK light truck of Beiqi Foton Motor loaded with 89995051 engine type of Foton Cummins and 6-speed transmission 6S500 gearbox of ZF Friedrichshafen as test vehicle. Input necessary data into model as basis of transformation. Basic data is shown in Tab.1

Table 1. Basic information of test vehicle.

\begin{tabular}{lc}
\hline Engine mode & turbo charging with inter-cooling \\
\hline Test mass & $8500 \mathrm{~kg}$ \\
Rated power & $115 \mathrm{kw}$ \\
Rated engine speed & $2950 \mathrm{rpm}$ \\
Idle engine speed & $750 \mathrm{rpm}$ \\
Dynamic rolling radius & $0.432 \mathrm{~m}$ \\
\hline
\end{tabular}

Transmission data including number of forward gears, gear ratios, final driver ratio and road load coefficients for testing is also inserted into the model.

\subsection{Characteristic engine speeds}

The external characteristic curve of the engine defines engine condition at full load under certain engine speed and thus is always a reliable basis for gear selection ${ }^{[4]}$.

Input engine's EMP test result into model, after proper processing, get its external characteristic curve. As showed in Fig.2:

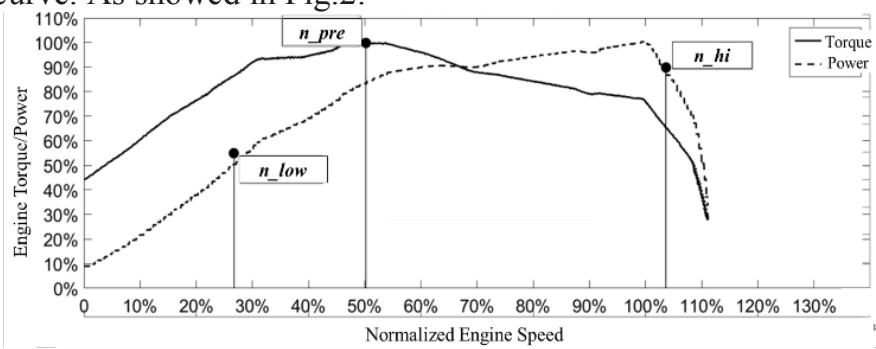


Fig. 2. External characteristic curve of test engine.

Apply interpolation method to get three important characteristic engine speed values from the curve as the base of further gear use calculation.

$n_{l o}$ : lowest engine speed where the engine produces $55 \%$ of rated power at full load.

$\boldsymbol{n}_{\text {pre: }}$ : minimum engine speed where the engine torque reaches maximum.

$\boldsymbol{n}_{\boldsymbol{h}}$ : highest engine speed where the engine produces $90 \%$ of rated power at full load.

Normalized characteristic values of the test engine is calculated and showed in pop-up window in Fig.3.

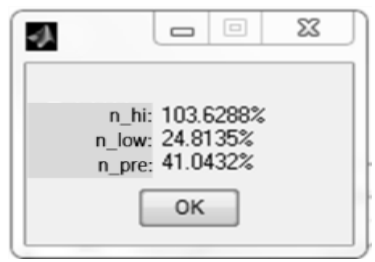

Fig. 3. Characteristic values of the test engine.

\subsection{Test vehicle cycle}

Vehicle cycle only changes with huge changes in traffic conditions and is thus stable over long periods of time. However, engine cycle changes significantly with engine and drive train technology and cannot be considered stable. This makes it unreliable and improper to construct engine cycle directly from engine data collected from real-life operation.

To better meet the various engine test scenarios and adapt to technique development and standard change, engine cycle should be constructed by transformation of a more stable vehicle cycle ${ }^{[5]}$.

For comparison with present standard cycle in next step, demonstration transformation was carried out using WHTC's underlying vehicle cycle WTVC in term of velocity-time $(V(t))$.

Corresponding vehicle power-time cycle $P(t)$ is also necessary input for transformation. $P(t)$ is calculated on base of $V(t)$ and test vehicle data. Calculation formula is below:

$$
P(t)=f_{0} \times V(t)+f_{1} \times V(t)^{2}+f_{2} \times V(t)^{3}+T M \times K R \times V(t) \times \frac{V(t)-V(t-1)}{3.6}
$$

where:

$P(t)$ : power pattern of vehicle cycle, $\mathrm{kw}$

$V(t)$ : velocity pattern of vehicle cycle, $\mathrm{km} / \mathrm{h}$

$T M$ : test mass of vehicle

$K R$ : inertial resistances of the drivetrain

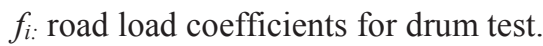

\subsection{Gear selection}

For each forward gear, based on test vehicle's transmission data, transform $V(t)$ to actual engine speed -time cycle $N e(t)(\mathrm{rpm})$. Calculation formula is below:

$$
N e(t)=\frac{V(t)}{2 \pi r} \times \frac{1000}{60} \times i_{m} \times i_{f}
$$

Where:

$i_{m}$ : gearbox transmission ratio of certain gear 
$I_{f}$. final driver ratio

$\mathrm{r}$ : dynamic rolling radius of test tire $(\mathrm{m})$.

Normalize $N e(\mathrm{t})$ with rated speed $N e_{\text {rated }}(\mathrm{rpm})$ and Idling speed $N e_{\text {idle }}(\mathrm{rpm})$ of the test engine:

$$
\mathrm{Ne}_{\text {norm }}(\mathrm{t})=\left(\mathrm{Ne}(\mathrm{t})-\mathrm{Ne}_{\text {idle }}\right) /\left(\mathrm{Ne} \text { 额定 }-\mathrm{Ne}_{\text {idle }}\right)
$$

For each forward gear, look up the external characteristics curve to get the maximum output power $P_{\max }\left(N e_{\text {norm }}(t)\right)$ according to its normalized engine speed $N e_{\text {norm }}(t)$.

Combined with calculated results above, apply model's built-in gear use strategy to calculate gear use second by second:

Practicability: The speed range between idling and $n_{l o}$ is only used when starting or gearshifts. $n_{l o}$ and $n_{h i}$ define the engine speed range for real-life operation. $N e_{n o r m}(t)$ under output gear should lie in this range;

Drivability: Make sure that engine can provide enough power for vehicle operation under output gear: $P_{\max }\left(\mathrm{Ne}_{\text {norm }}(t)\right)$ is higher than or equal to $P(t)$.

Adaptability: From gears fulfilling rules above, $G_{\text {model }}(t)$ under which $N e_{\text {norm }}(t)$ is closest to $n_{\text {pre }}$ is selected for this second.

Calculate $G_{\text {model }}(t)$ second by second. The thin dotted line in Fig. 4 represents $\mathrm{G}_{\text {model }}(t)$ for urban part of WTVC.

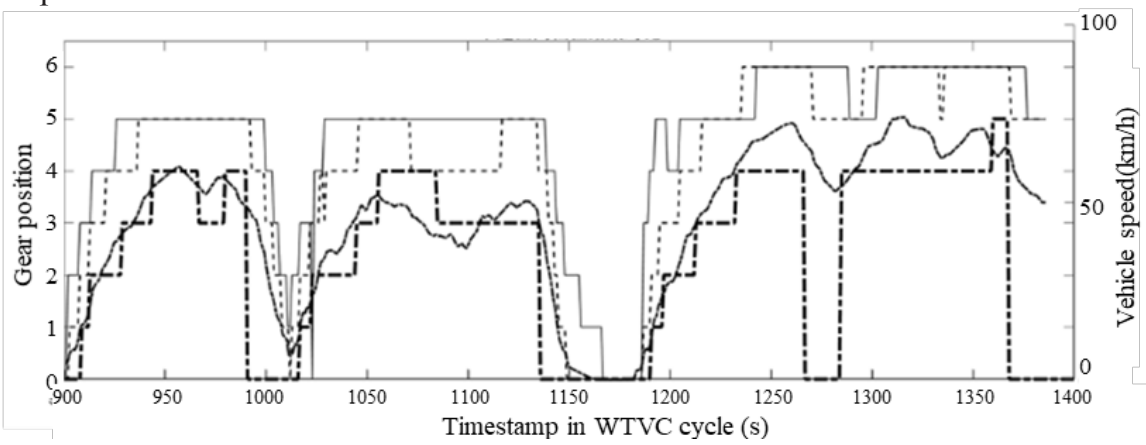

Fig. 4. Gear use for urban part of WTVC cycle.

\subsection{Engine cycle construction}

Load-time cycle $L e(t)$ is calculated by dividing vehicle power by engine max power under $G_{\text {model }}(t)$; as showed in the equation below:

$$
\operatorname{Le}(t)=\frac{P(t)}{P_{\max }\left(N e_{\text {norm }}(t)\right)}
$$

At last, output test vehicle's engine cycle termed by $N e_{n o r m}(t)$ and $L e(t)$. In Fig.5, $V(t)$ of underlying WTVC cycle is represented by the dot dash line; $N e_{n o r m}(t)$ is represented by thick solid line; $L e(t)$ is represented by thin solid line. 


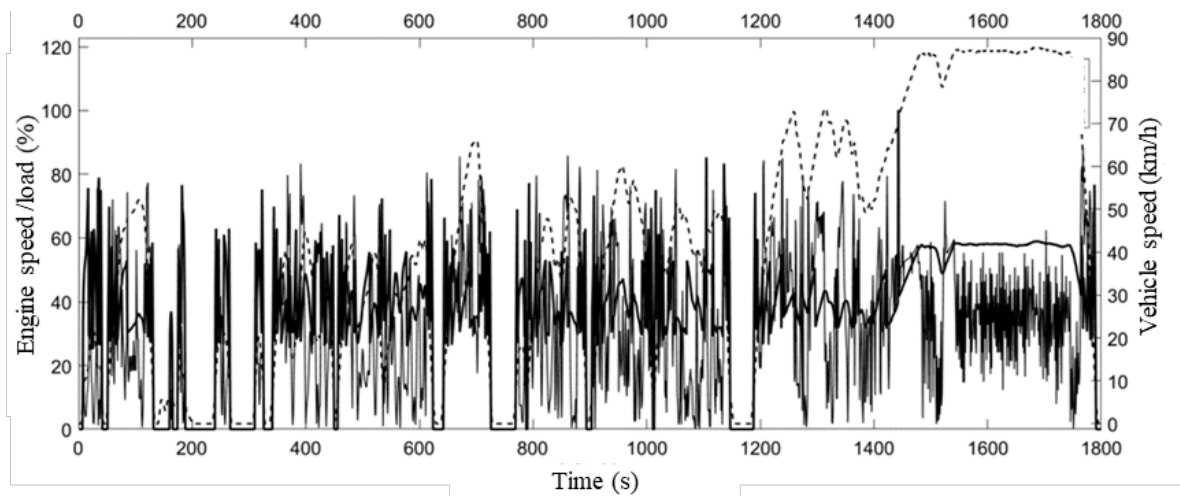

Fig. 5. Output engine cycle for WTVC.

Joint distribution of the two cycle parameters is showed in Fig. 6.

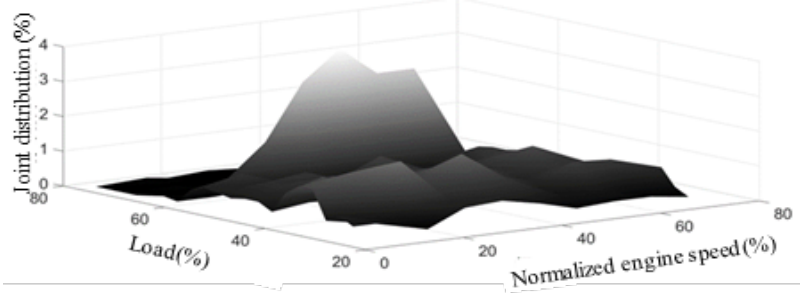

Fig. 6. Joint distribution of engine cycle parameters.

\section{Cycle transformation example}

To verify the rationality and reliability of output engine cycle (hereinafter referred to as 'model cycle'), compare it with WHTC engine cycle used in N6, which is also transformed from WTVC.

\subsection{Gear information for Cycle}

WHTC provides a reference cycle of normalized engine speed $N e_{w}(\mathrm{t})$, and calculation formulas to transform it to a customized engine speed (rpm) cycle for actual test, as showed below:

$$
\mathrm{Ne}_{\text {test }}(\mathrm{t})=\mathrm{Ne}_{\mathrm{w}}(\mathrm{t}) \times\left(0.45 \times \mathrm{n}_{\text {lo }}+0.45 \times \mathrm{n}_{\text {pre }}+0.1 \times \mathrm{n}_{\text {hi }}-\mathrm{Ne}_{\text {idle }}\right) \times 2.0327+\mathrm{Ne}_{\text {idle }}
$$

Calculate $N e_{\text {test }}(\mathrm{t})$ for test engine; plot scatter gram with $V(t)$ of WTVC, as showed in Fig.7: 


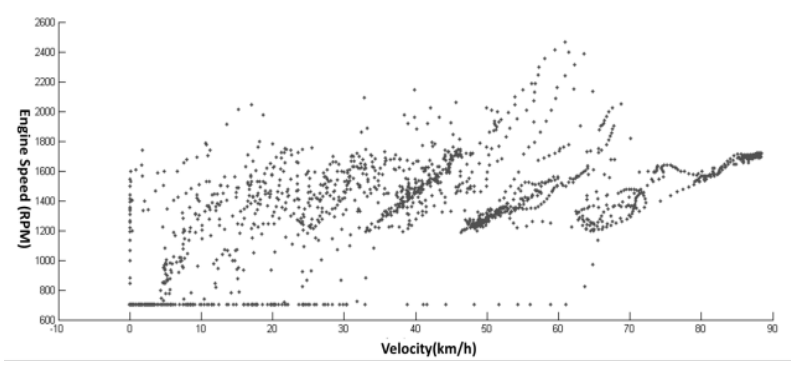

Fig. 7. WTVC vehicle speed - WHTC engine speed.

There are 6 distinct linear relations between $N e_{\text {test }}(t)$ and $V(t)$, indicating that transformation from WTVC to WHTC is based on a 6-speed transmission gearbox model, which is in line with test vehicle.

Ratio between $N e_{\text {test }}(t)(\mathrm{rpm})$ and $V(t)(\mathrm{km} / \mathrm{h})$ (hereinafter referred to as ' $N V_{\text {test }}{ }^{\text {') }}$ under each gear can be sorted out by clustering algorithm, as showed in the left column of Tab. 2.

Table 2. $\quad V$ for each gear.

\begin{tabular}{lcc}
\hline & $N V_{\text {test }}$ & $N V_{\text {actual }}$ \\
\hline Gear 1 & 121.77 & 120.00 \\
Gear 2 & 80.01 & 80.47 \\
Gear 3 & 54.70 & 55.51 \\
Gear 4 & 38.38 & 38.02 \\
Gear 5 & 27.73 & 28.69 \\
Gear 6 & 18.78 & 23.07
\end{tabular}

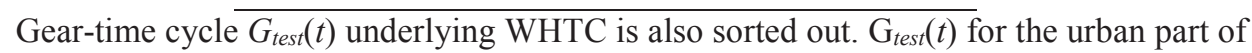
WHTC is represented by solid line in Fig. 4.

\subsection{Comparison between cycles}

From top to bottom, Fig.8 compares $N e(t)$ of model cycle and $N e_{\text {test }}(t)$ of WHTC corresponding to urban, rural and motorway section of WTVC. Solid line stands for model cycle while dot line for WHTC.

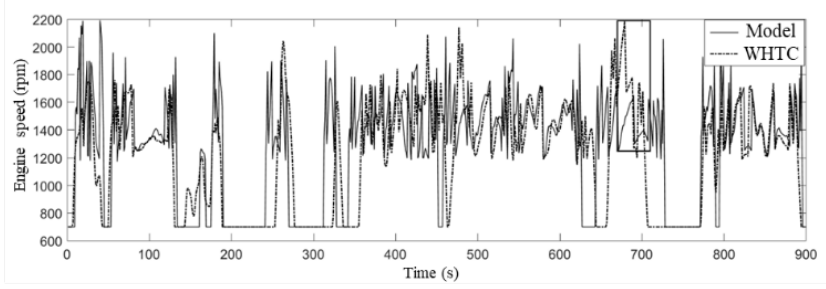

a) Urban section 


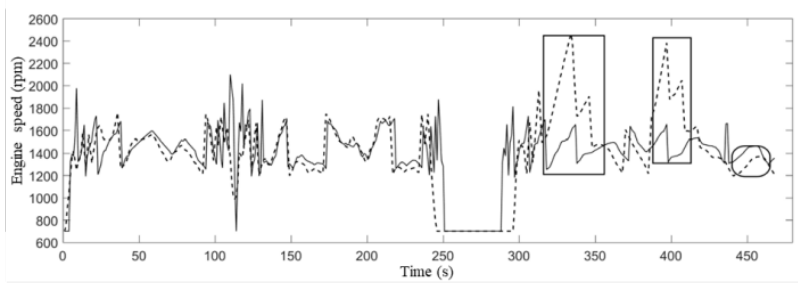

b) Rural section

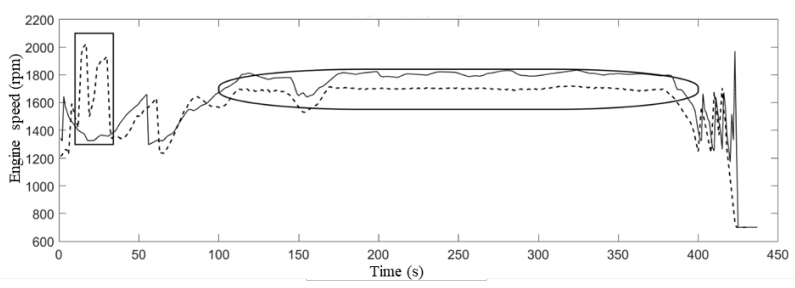

c) Motorway section

Fig. 8. Engine speed of two cycles.

The two cycles are highly identical for most of time, indicating that underlying technical routine of the method is in good agreement with present standard, and its model operation is quite reliable.

However, obvious discrepancy exists in few time periods. Extract these periods and analysis the reason underlying the discrepancy.

(1) Discrepancies generated by gear selection

4 time periods are outlined by squares in Fig. 8: 670-710s of rural section, 320-350s and $400-420$ s of urban section, $10-30$ s of motorway section. In these periods, structural discrepancy indicates significant gap between $N V$ of two cycles, which is generated by gear selection divergence under same $V(t)$. lower $N e(t)$ of model cycle implies higher gear use.

Extract corresponding time periods of WTVC: these periods represent acceleration phase under relative high vehicle speed. Under the preconditions to meet the acceleration power demand, model is inclined to upshift earlier, which represents a more economic gear use strategy ${ }^{[6]}$.

(2) Discrepancies under same gear selection

2 time periods are outlined by ovals in Fig. 8: 440-480s of rural section and 100s-400s of motorway section. In these periods, obvious but unstructured discrepancy between two cycles indicates different $N V$ under the same gear.

Extract the corresponding gear-time cycle: it is observed that these periods are all under $6^{\text {th }}$ gear use.

Get the test vehicle' actual ratio between engine and vehicle speed $N V_{\text {actual }}$ under each gear and compare it with $N V_{\text {test }}$, as showed in Tab.2. It is observed that unlike other gears, significant gap exists between two ratios under $6^{\text {th }}$ gear and explains the speed discrepancy.

Ratio used for model transformation is calculated based on inputted actual test vehicle information and is naturally closer to $N V_{\text {actual. }}$. This results in the higher output $N e(\mathrm{t})$.

Compared with WHTC, detailed input data features the model the highest representativity for engines of different technologies and test scenarios. Thus, model cycle accords better to real-life engine condition of the corresponding vehicle drum test.

\subsection{Comparison with drum test cycles}

Compare WTVC vehicle drum test result with model output under the same test setting. 
Gear selection of heavy-duty vehicle drum test is left to driver's own discretion. Thick dotted line in Fig. 4 represents gear-time cycle in this test. Divergence from model output is quite obvious: driver is more likely to select lower gears in drum test.

Fig.9 shows the speed-load output of model and drum test, which is significantly influenced by gear use divergence: With higher gear, engine speeds of the model cycle are generally higher; load distribution is much higher in the more efficient zone of $80-100 \%$.

Under the precondition to satisfy power demand, as for model's gear use strategy, engine generally operates with higher efficiency, thus minimize fuel consumption and emission ${ }^{[7]}$.

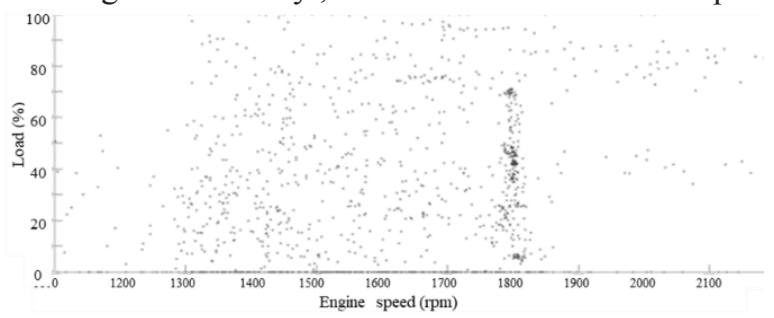

a) Model cycle

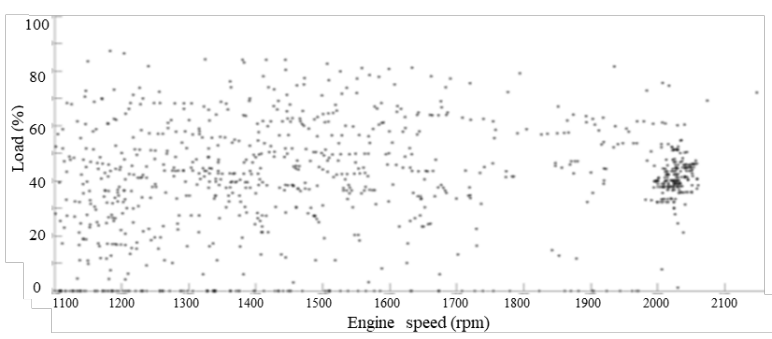

a) Drum test

Fig. 9. Engine speed-load.

\section{Conclusions}

This paper presents a technical method to derive engine cycle by constructing a vehicle-toengine cycle transform model. vehicle cycle and test vehicle data is inputted, processed and used to get engine condition under each gear; Apply built-in gear use strategy to calculate gear use; Under selected gear, transform vehicle cycle into engine cycle in terms of normalized speed and load.

Use WTVC cycle and typical test vehicle to demonstrate the transformation method and model. Compare output cycle with present standard WHTC cycle and WTVC drum test drum result.

Firstly, comparison result proves the model's consistency with present standard and reliability of its model operation; Secondly, model can generate customized engine cycle adapted to various test settings and according to real-life engine test condition; Finally, gear use strategy adopted by the model is featured with economy, resulting in better consumption and emission test results.

In the long run, this method can meet the requirements of technological progress and regulatory changes, and provide technical support for the government, research institutions and enterprises in the field of heavy vehicle engine related policy-making, test design and product development. 


\section{References}

1. GB/T 27840-2011 Fuel consumption test methods for heavy-duty commercial vehicles

2. Chen Lin J. Study on the Emission and Test Methods of Vehicle Compared with Diesel Engine Bench Based on CHINA VI Emission Standard Heavy-duty Vehicles. (Doctor Thesis) Jiangsu University, Zhenjiang, Jiangsu, China, 2018.

3. Yu Hongfeng J. The Study of Dynamic Emission Simulation Method of Turbocharged Engine. (Master Thesis) Wuhan University of Technology, Huhan, Hubei, China, 2006.

4. Liu Jingping., Li Yingchun., Xia Xiaolang., A Study on the Determination of Simplified Engine Operation Points Corresponding to Vehicle Driving Cycle, Automotive Engineering: 564-569, 2011(7)

5. Heinz Steven, Development of a Worldwide Harmonized Heavy-duty Engine Emissions Test Cycle Final Report,

6. Li Jian., Yao Jianmin., Wu Xuling., Research on Vehicle WLTC Fuel Consumption Based on Engine Steady Working Conditions, Chinese Internal Combustion Engine Engineering: 235-240, 2016(6)

7. Zhao Guobin., Ge Yongtian,Geng Shuai1., Jin Ling., . Experimental Comparison and Study of WHSC/WHTC and ESC/ETC Test Cycles, Chinese Journal of Automotive Engineering: 29-34, 2015 (1) 